\title{
New interpretation of the boundary diffracted wave origin
}

\author{
S.P. Anokhov \\ International Center "Institute of Applied Optics" of NAS of Ukraine
}

\begin{abstract}
The important improvement for Young's model of diffraction is proposed. This interpretation is based on the statement about the existence of the energy transfer process directed from the remaining field towards the boundary wave, that is formally taken into account by introduction of a source for this wave on boundary of a geometrical shadow. The legitimacy of the application, in this case, of parabolic equation in the form of the standard equation of diffusion - thermal conductivity is validated. With the help of such approach it was obtained the compact analytical representation of this wave at the diffraction of a plane wave on a half-plane, which is completely concordant with Sommerfeld's rigorous solution and available experimental facts.
\end{abstract}

Keywords: diffraction, boundary wave, half-plane, parabolic equation, boundary of a geometrical shadow.

Paper received 05.10.99; revised manuscript received 22.12.99; accepted for publication 21.03.00.

\section{Introduction}

The evolution of the diffraction theory associated with the substitution of fictitious secondary wave sources of Hugens-Fresnel by the pair of real waves of YoungSommerfeld-Rubinowicz [1,2] had almost no effect on practical optics where the customary Kirchhoff's theory is still dominating. This can be explained, in particular, by abnormality of the boundary wave physical properties that are evidently exceed the limits general to all traditional types of waves. (Wave abnormalities of the similar kind have just recently become the subject of specialists' interest. In this way the development of the so called singular optics was initiated.) The boundary wave appearance mechanism is the most enigmatic, and its interpretation since Young did not pass further his hypothesis on "a kind of reflection".

The goal of the present paper is to validate a simple and, according to author's point of view, the most natural version of the boundary wave appearance which is free from previous logical impediments and makes a new statement of the diffraction problem possible. A particular solution of the problem for a half-plane coincides with the Sommerfeld rigorous one but can be obtained by simpler mathematical means.

\section{Logical prerequisites}

Following Sommerfeld [1,2], let us consider the diffraction field as an aggregate of two wave components, namely: geometrical, $A_{G}$, the remaining part of initial wave that is subjected to a discontinuity at the edge of the screen and propagates further according to the geometrical optics law; the second - boundary $A_{B}$ - cylindrical wave, the geometrical center of which coincides with the edge of half-plane (Fig. 1). The glow of a real screen edge that corresponds to this wave can be easily observed in experiments, but its true origin is still unknown.

Indeed neither reflection nor scattering of the incident wave at the screen edge may cause it. This follows even from the well established fact - neither reflectivity of an obstacle material nor radius of curvature nor quality of its edge finishing influence the diffracted field [1]. Artificial introduction of scattering, for example, by making the edge surface coarse, results just in general blurring of the observed pattern [3]. Furthermore, the specific spatial character of the boundary wave itself can be scarcely associated with any known phenomenon of this kind.

Years of unsuccessful search of the boundary wave origin among known phenomena point out on the necessity to introduce some qualitatively new hypothesis. However, as far as the author knows, the problem has not been stated in a similar way yet. A recurrent decisive 


\section{S.V. Anokhov. New interpretation of the boundary diffracted wave origin.}

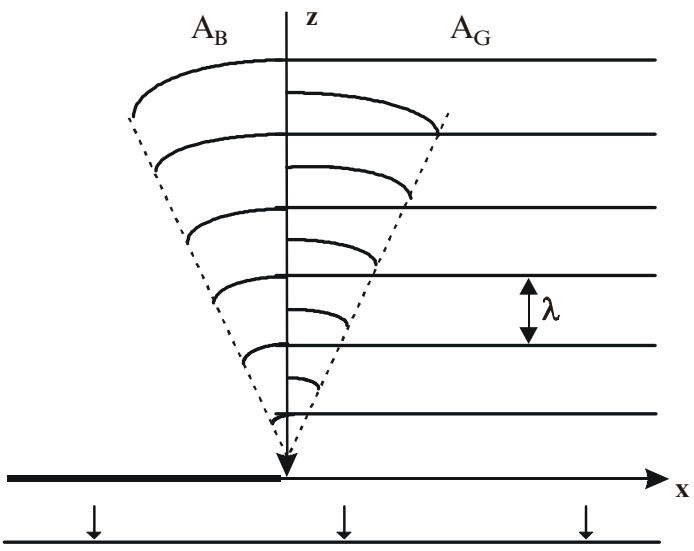

Fig. 1. Schematic explanation of diffraction field structure behind the screen edge based on rigorous diffraction theory.

attempt to find in the boundary wave field any confirmation of its interaction with a screen [4] that is almost considered as the criteria of physical validity of Young's concept in general, may serve as an example of a contemporary approach.

Explanation of the boundary wave power loss compensation during its spatial spread remains very difficult in this concept. As is well known, during a plane wave diffraction at a half-plane the field amplitude at the shadow boundary equals to the half of incident wave amplitude regardless a specific position of the control plane [2,5]. This circumstance being quite obvious in Fresnel's zone approach has no satisfactory explanation after crossing over Young's model. Moreover, any attempt to explain the situation logically becomes here difficult without an assumption on continuous energetic replenishment of the boundary wave.

The legitimacy of this assumption is also confirmed by general evolution of the field formed at aperture diffraction. Really, in immediate proximity to the aperture practically entire wave energy penetrating through it evidently belongs to the geometrical component of the field. On the contrary, in the far field the prevalent part of this energy is distributed beyond the limits of the geometrical shadow where the presence of just boundary waves is possible. As is obvious, there are all grounds to connect real evolution of the diffraction field with the existence of the directional energy transfer from geometrical component towards boundary waves.

\section{Hypothesis}

To originate a description of the diffraction that would non-contradictory combine all facts referred above, it is sufficient to suppose that the real cause of the boundary wave appearance is the discontinuity of wave field at the edge of the obstacle. In the absence of appropriate physical grounds, this cause may be determined as the rule of inadmissibility of sudden changes for the intensity of free electromagnetic wave. Action of this rule does not contradict to the laws of electrodynamics and also matches to all other existing requirements to field strength in nonuniform space, for instance, to the continuity of its tangential component or to the conservation of normal component normalized onto the media dielectric constant.

As a result, any wave front discontinuity leads to the immediate conversion of its new edge into the compensating wave field which realizes required smoothing of originated sudden change in field strength. The ground for the hypothesis is the extremely irregular structure of the boundary wave itself which always is strictly concordant to specific parameters of the discontinuity. The only known situation in optics where such a rule may be realized is just the diffraction that is originated by partial bordering of a free wave. In this approach the real source of the observed glow is the wave front discontinuity which is adjoined with the screen edge at the initial moment.

Thus, the originated front cut is conserved and boundary wave inevitably diverges - field discontinuity compensation becomes incomplete at once. Therefore, the energy inflow from the geometrical wave into the boundary one does not cease, moreover it becomes a continuous process directed towards the keeping of required compensating field amplitude. This process explains the observed energy replenishment of the bounrdary wave. The field amplitude remains constant due to the dynamic equilibrium between energy outflow and inflow in the discontinuity area.

Taking into account all stated above, the formation of the boundary wave should be interpreted as the specific phenomenon caused by the ability of the electromagnetic field to realize immediate auto-compensation of imposed to it discontinuity. As the name of the phenomenon, it is proposed to use the term «wave conversion». Already mentioned difference of this phenomenon from traditional wave processes (where transformation of one wave into another is possible only at their nonlinear interaction) becomes unimportant when considering the proposed model, similarly to Huygens-Fresnel's model of secondary waves, only as a mode of argumentation which is not pretending to intrinsic "material" reliability.

Nevertheless, the problem of real existence of such process remains unsolved. As the geometrical component is not a wave in the conventional sense of this word and can not be selected experimentally, it is hardly to logicaly estimate its donor function from usual wave positions. Therefore, it is impossible to consider the groundless statement about the action of real energy redistribution process inside the diffraction field. The serious reason for the benefit of this version is the fact of successive growth of the boundary wave energy observed in the experiment.

\section{Mathematical means}

The proposed interpretation has many common features with the existing model of the diffraction process based on its analogy with well known in optics and acoustics 


\section{S.V. Anokhov. New interpretation of the boundary diffracted wave origin.}

phenomenon of wave amplitude transverse diffusion [6]. The advantages of such approach towards the diffraction were shown for the first time for solving the problem of radio-waves distribution above the surface of the Earth [7,8] and, later, during the development of open cavities' theory [9]. It is talked about the application of the parabolic equation

$2 k i \frac{\partial A}{\partial z}=\frac{\partial^{2} A}{\partial^{2} x}+\frac{\partial^{2} A}{\partial^{2} y}$,

obtained from wave one through its application towards weakly divergent waves in the form of

$$
U(x, y, z, t)=A(x, y, z, t) e^{i(\omega t-k z)},
$$

where the amplitude $A(x, y, z)$ is a slow changing (in the scale of a wave length 1) function of coordinates, and the direction of wave propagation coincides with axis $z$.

The transition from the wave equation to its much simpler paraxial variant has appeared completely proved just for the diffraction, where all major wave processes are concentrated near the boundary of the geometrical shadow, i.e. near the chosen direction of field propagation. (Other important class of problems for this equation has appeared recently in connection with the study of wave beams [10]).

The possibility to use the parabolic equation for the description of the boundary wave is based on its quite clear paraxial character. The computer analysis of the Sommerfeld solution shows that up to $90 \%$ of this wave's energy is focused in angular sector $\theta \leq 6(\lambda / 2 L)^{1 / 2}$, whose bisectrix is the boundary of the geometrical shadow (Fig.1). It is easy to estimate that in visual range of spectrum $(\lambda=600 \mathrm{~nm})$ the indicated angle does not exceed two degrees for the distance $L=1 \mathrm{~cm}$ from the edges of the halfplane, and equals to twelve angular minutes for $L=1 \mathrm{~m}$.

Let us note, that the accuracy of the paraxial approximation at description of the diffraction process is noticeably reduced only in immediate proximity to the edges of the obstacle $\left(L \leq 10^{3} \lambda\right)$, where the directional pattern of the boundary wave is essentially broaden. However, this correction has no practical value for calculations as the most part of the electromagnetic energy in this area still belongs to the geometrical component.

If one assumes that $z=c t$, i.e. chooses the frame of reference moving with the speed of light along the $z$ axis (the beginning of which at the moment $t=0$ coincides with the plane $z=0$ ), the equation (1) becomes one of the diffusion- thermal conductivity, well known in mathematical physics $[6,11]$ :

$$
\frac{\partial A}{\partial t}=D\left(\frac{\partial^{2} A}{\partial^{2} x}+\frac{\partial^{2} A}{\partial^{2} y}\right)
$$

where $D=\lambda c / 4 \pi i$. The transition from the stationary equation (1) to the dynamic one (3) is possible to interpret as the returning to the presentation of propagating initial wave (2), but with the relocation in two-dimensional transversal system of coordinates $(x, y)$, which moves together with the wave.

The indicated replacement formally completely equalizes the considered process with well-studied diffusion and thermal conductivity phenomena. The imaginary value of $D$ coefficient in this case only means that in contrast to the diffusion of material particles the referred process has wave nature. The phenomenon described by the equation (3) consists in transversal drift of wave amplitude revealing itself in gradual smoothing of any wave amplitude gradient at the cost of its energy transfer along the wavefront [6]. As an example of this process, the well known transversal spread of restricted wave beams could serve.

\section{Diffraction of a plane wave on a half-plane}

For approbation of the new approach, we took the wellknown problem of a plane wave diffraction on a halfplane. Existence of Sommerfeld's rigorous solution [1,2] makes it possible to consider this problem as a test. First let us solve it in a general form, without attracting of Young's model. We shall consider normal incidence of a simple plane harmonic wave onto the arbitrary screen with the rectilinear edge (Fig. 1). If one combines this edge with the $y$ axis, and the perpendicular of the plane wave - with the $z$ axis, the problem of the diffraction is reduced to search of amplitude distribution $A(x, t)$ that would satisfy the equation (1) without the second member in its right part and initial condition

$$
A(x, 0)=\left\{\begin{array}{lll}
A_{0} & \text { for } & x \geq 0 \\
0 & \text { for } & x<0
\end{array}\right.
$$

In the frame of reference connected with the propagating wave, the considered problem represents one-dimensional diffusion process in unbounded limits (the Cauchy problem): $-\infty<\mathrm{x}<\infty, t>0$. Its solution is well known [11]:

$$
A(x, t)=\frac{A_{0}}{2}\left[1+\Phi\left(\frac{x}{2 \sqrt{D t}}\right)\right]
$$

where $\Phi(z)=\frac{2}{\sqrt{\pi}} \int_{o}^{z} \exp \left(-\alpha^{2}\right) d \alpha$ is the error integral, and for unity of length, depending on $t$, starts value $2 \sqrt{D t}$. As $F(0)=0$, it immediately follows from (5) that the field amplitude on the boundary of the geometrical shadow $(x \equiv 0)$ is identical to $A_{0} / 2$, i.e. equal to the halfsum of their values on the left and the right sides of the discontinuity point coordinate that is usual for diffusion problems. 


\section{S.V. Anokhov. New interpretation of the boundary diffracted wave origin.}

Taking into account that the error integral is simply bounded to the Fresnel integrals (for example, [12]):

$$
\Phi\left(v \sqrt{\frac{i \pi}{2}}\right) / \sqrt{2 i}=C(v)-i S(v)
$$

where, $C(v)=\int_{0}^{v} \cos \left(\frac{\pi}{2} t^{2}\right) d t, S(v)=\int_{0}^{v} \sin \left(\frac{\pi}{2} t^{2}\right) d t$, the general distribution of the diffraction field (5) can be presented by expression

$$
A(v)=\frac{A_{0}(1+i)}{2}\left\{\left[\frac{1}{2}+C(v)\right]-i\left[\frac{1}{2}+S(v)\right]\right\},
$$

in which $u=x \sqrt{2 / \lambda L}$, and $L=c t$ is a distance from the observation plane to the diffraction one.

Accordingly, the intensity of this field is characterized by expressions $J=A \cdot A *$ or

$$
J(v)=\frac{A_{0}^{2}}{2}\left\{\left[\frac{1}{2}+C(v)\right]^{2}+\left[\frac{1}{2}+S(v)\right]^{2}\right\},
$$

reproducing the field distribution that had already become canonical. In this case, both distributions Kirchhoff's and Sommerfeld's - completely coincide [2]. Thus, the legitimacy of application of the non-stationary parabolic equation (3) for solving similar problems gains convincing confirmation.

\section{Conclusion}

Thus, with the separation of the diffraction field on "Young" components - the geometrical one and the boundary wave- we face the objective necessity of introduction (at least, formal) of the energy transfer process directed from the first wave component towards the second. It is possible to have skeptic attitude towards the idea of wave conversion, but the self-coherence of the proposed model, eliminating practically all logic difficulties of Young's approach can hardly be denied. At the same time, all advantages of this approach reducing the diffraction process to the interaction of two standard wave components, evolving according to standard algorithms, are maintained.

\section{References}

1. A. Sommerfeld, Optics, Academic Press, NY, 1954.

2. M. Born, E. Wolf, Principles of Optics, 6-th ed., Pergamon Press, Oxford, 1991.

3. G.S. Gorelik, Oscillations and waves, Moscow, GIFML, p.572, 1959 (in Russian)

4. P. Langlois, A. Boivin, Thomas Young's ideas on light diffraction in the context of electromagnetic theory // Can. J. Phys. 63, pp. 265-274, 1985.

5. J. D. Barnett, F. S. Harris, Test of the effect of edge parameters on small-angle Fresnel diffraction of light at a straight edge // J. Opt. Soc. Am. 52, pp. 637-643, 1962.

6. G.D. Malyuzhinets, Progress in concepts of diffraction phenomena // Uspekhi Fizich. Nauk, 69, pp. 321-334, 1959 (in Russian).

7. M.A. Leontovich, About a method for solving of the problem of electromagnetic waves propagation along the surface of the Earth // Izvestiya AN SSSR, ser. Phys., 8, pp. 16-22, 1944 (in Russian).

8. M.A. Leontovich, V.A. Fok, Solution of the problem of electromagnetic waves propagation along the surface of the Earth with the help of parabolic equation method // Zhurn. Exper. $i$ Teor. Fiz., 16, pp. 557-573, 1946 (in Russian).

9. L.A. Vainshtejn, Open resonators and open waveguides, Moscow, Sovetskoe radio, p. 476, 1976 (in Russian).

10. H. Kogelnik, T. Li, Laser Beams and Resonators // Appl. Opt., 4, pp. 1562-1569, 1965.

11. A.N. Tikhonov, A.A. Samarskij, Equations of mathematical physics, Moscow, Nauka, 724 p., 1966 (in Russian).

12. A. Ango, Mathematics for electro- and radioengineers, Moscow, Nauka, p. 780, 1965 (in Russian). 